\title{
Population III X-Ray Binaries
}

\section{Taeho Ryu*, Takamitsu L. Tanaka and Rosalba Perna}

The State University of New York, Stony Brook, USA

E-mail: taeho.ryuestonybrook.edu

Understading of the role of X-rays for driving the thermal evolution of the intergalactic medium (IGM) at high redshifts is one of important questions in astrophysics. High-mass X-ray binaries (HMXBs) in early stellar populations are prime X-ray source; however, their formation efficiency is not well understood. Using $N$-body simulations, we estimate the HMXB formation rate via mutual gravitational interactions of nascent, small groups of the Population III stars. We find that HMXBs form at a rate of one per $\gtrsim 10^{4} \mathrm{M} \odot$ in newly born stars, and that they emit with a power of $\sim 10^{41} \mathrm{erg} \mathrm{s}^{-1}$ in the $2-10 \mathrm{keV}$ band per star formation rate (SFR). This value is a factor $\sim 10^{2}$ larger than what is observed in star forming galaxies at lower redshifts; the X-ray production from early HMXBs would have been even more copious, if they also formed in situ or via migration in protostellar disks. Combining our results with earlier studies suggests that early HMXBs were highly effective at heating the IGM and leaving a strong $21 \mathrm{~cm}$ signature. We discuss broader implications of our results, such as the rate of long gamma-ray bursts from Population III stars and the direct collapse channel for massive black hole formation.

Frontier Research in Astrophysis - II

23-28 May 2016

Mondello (Palermo), Italy

\footnotetext{
* Speaker.
} 


\section{Introduction}

One of the fundamental challenges in cosmology is to understand the history of the early Universe from Cosmic dark ages to the end of reionization as the first stars and galaxies emerged and the radiation from these objects had ionized the intergalactic medium (IGM). Advances in numerical techniques, combined with exquisite measurements of the "initial" conditions ([1], [2]), have led to remarkable simulations ([3], [4], [5], [6], [7]) of the conditions leading up to the former milestone, occurring at $z \gtrsim 30$, when the Universe was $\approx 100$ Myr old. However, reconstructing the subsequent several hundred Myr of cosmic history has embraced difficulties, one of which is related to uncertainties in modeling the numerous forms of feedback from the first astrophysical objects ([8], [9], [10]).

In particular, X-rays from the first galaxies can act as a powerful source of feedback ([11], [12]). Because hard X-rays (energies $\gtrsim 1 \mathrm{keV}$ ) have mean free paths comparable to the Hubble horizon, they can isotropically heat and partially reionize the early IGM ([13], [14], [15], [16]). Recent studies show that they are expected to be the dominant agent in heating the IGM, possibly leading to suppression of star formation ([17]) and massive black hole (BH) growth ([18]) inside low-mass dark matter haloes by raising the Jeans and filtering masses of the IGM ([19], [20]). On larger scales, as soft X-rays $(\sim 0.1-1 \mathrm{keV})$ promotes the formation of molecular hydrogen, affecting the formation of stars and possibly massive black holes (BHs) ([21], [22], [23], [24]).

The goal of this study is to estimate the formation rate and X-ray output of HMXBs in the early Universe as one of the prime $\mathrm{X}$-ray sources, by using $N$-body simulations of nascent groups of the first (Population III, henceforth Pop III) stars. The formation rate has been left as a free parameters in studies or estimated with simplified assumptions. However, we estimate this value via purely theoretical dynamical approach. Based on the estimated formation rates, we derive a HMXB energy output (normalized to the star formation rate) that is a factor $\sim 10-150$ higher than in present-day star-forming galaxies. We find that the X-ray output does not change significantly within the wide variety of simulation setups and submit that this is a robust estimate. Our work is also relevant for predicting the rates of long-duration gamma-ray bursts (LGRBs) from Pop III stars.

The paper is organized as follows. We start in $\$ 2$ by discussing the problem to be solvedbeginning with the equations of motion, followed by the description of our $\mathrm{N}$-body code, our choices for the initial conditions, and how the data is interpreted for HMXB formation. We present our results in $\S 3$. In $\S 4$, we discuss the implication of our work for the X-ray output of the first galaxies, as well as for other topics such as LGRBs and SMBH formation. We conclude with a summary of our findings in $§ 5$.

\section{Stellar Dynamics}

Here, we provide an overview of our simulations-namely: the equations of motion that are solved to simulate the dynamical evolution of the star groups; the numerical scheme we use to solve the equations; the different types of initial conditions we adopted, as well as the reasoning behind our choices; and finally, how the results are interpreted for HMXB formation. 


\subsection{The equations of motion}

Our $N$-body code numerically solve the equation of the motion of $N$ objects with masses $m_{i}$, moving under their mutual gravitational influence, a dissipative dynamical friction force, and a background gravitational potential, which can be written as,

$$
\frac{d^{2}}{d t^{2}} \vec{r}_{i}=\vec{a}_{\mathrm{g}, i}+\vec{a}_{\mathrm{df}, i}+\vec{a}_{\mathrm{bg}, i}
$$

The first term on the right-hand side of equation (2.1) is the specific force due to Newtonian gravity,

$$
\vec{a}_{\mathrm{g}, i}=-\sum_{j \neq i} G m_{j} \frac{\partial S\left(r_{i j}\right)}{\partial r_{i j}} \frac{\vec{r}_{i}-\vec{r}_{j}}{r_{i j}},
$$

where $G$ is the gravitational constant, $\vec{r}_{i}$ is the displacement of the $i_{\text {th }}$ star from the center of the host dark matter halo, and $r_{i j} \equiv\left|\vec{r}_{i}-\vec{r}_{j}\right|$.

We adopt the Plummer softening kernel $S\left(r_{i j}\right)$ ([27]),

$$
S\left(r_{i j}\right)=-\frac{1}{\sqrt{r_{i j}^{2}+\varepsilon^{2}}},
$$

where we take $\varepsilon=R_{\odot}$.

The second term on the right-hand side of equation (2.1), $\vec{a}_{\mathrm{df}, i}$, is the specific drag force due to dynamical friction. For collisionless systems, the standard Chandrasekhar formula for dynamical friction is [27],

$$
\vec{a}_{i}=-4 \pi \ln \Lambda f\left(X_{i}\right) \frac{G^{2} m_{i}}{v_{i}^{3}} \rho\left(r_{i}\right) \vec{v}_{i},
$$

where

$$
f\left(X_{i}\right) \equiv \operatorname{erf}\left(X_{i}\right)-\frac{2}{\sqrt{\pi}} X_{i} \exp \left(-X_{i}^{2}\right)
$$

$v_{i}$ is the speed of the $i_{t h}$ star with respect to the background, $X_{i} \equiv v_{i} /\left(\sqrt{2} \sigma_{v}\right), \sigma$ is the velocity dispersion, $\ln \Lambda$ is the Coulomb logarithm and $\rho\left(\vec{r}_{i}\right)$ is the local gas density.

We adopt the modified formula for gaseous medium used in [28]. This prescription incorporates behaviors found in numerical simulations for subsonic and supersonic regimes ([29], [30]). The specific drag force vector always points opposite to the direction of motion. We use $\ln \Lambda=3.1$.

The third and last term, $\vec{a}_{\mathrm{bg}, i}$, is the specific force due to the background potential, which is dominated by gas. The background potential provides an additional inward force whose functional form depends on the density profile. For simplicity, here we use a constant density and explore different values in our simulations. The force due to the background potential is then

$$
\vec{a}_{\mathrm{bg}, i}=-\frac{4 \pi}{3} G \rho \vec{r}_{i},
$$

where here $\vec{r}_{i}$ is the vector pointing from the center of the halo to the $i$-th star.

The equation of motion is solved iteratively, with the positions, velocities and accelerations of each star updated at every time step. We describe our computational method below. 


\subsection{Code Description}

We perform 3-dimensional, $N$-body simulations with 4th-order \& 5-stage Runge-Kutta-Fehlberg methods (RKF45 method, [31]) using adaptive time steps. The RKF45 is a very precise and stable integration method among the large class of Runge-Kutta schemes, particularly by adapting the Butcher tableau for Fehlberg's 4(5) method.

The code keep updating the position and velocity by integrating the equations of motion (2.1), taking into account the effects of dynamical frictions and gravitational force from ambient gas.

To ensure numerical precision, our computational scheme varies the value of each subsequent time step analytically, so that numerical errors for each variable in the simulation do not exceed $10^{-13}$ times the size of the variable.

\subsection{Determining HMXB formation}

It is assumed that a HMXB has formed if both of the following criteria are satisfied:

1. One of two stars forming a binary turns into a compact object $(C O)$. We compare the typical lifetime $\left(\tau_{\text {life }}\right)$ of a massive star with the time $\left(t_{\text {run }}\right)$ in the simulation (taken to coincide with the time at which stars are born). If $\tau_{\text {life }}>t_{\text {run }}$, the star is marked as a CO in the simulation.

2. The two stars are close enough so that the accretion occurs through Roche-lobe overflow $(R L O F)$. This criterion is simply written as $R_{\mathrm{star}} \geq R_{\mathrm{RL}}$, where $R_{\mathrm{star}}$ is the stellar radius, and $R_{\mathrm{RL}}$ is the Roche-lobe radius of the most massive star calculated from the center of the star to the inner Lagrange point given by [59],

$$
\frac{R_{\mathrm{RL}, 1}}{r}=\frac{0.49 q^{2 / 3}}{0.6 q^{2 / 3}+\ln \left(1+q^{1 / 3}\right)}
$$

where $r$ is the orbital distance and $q=\frac{m_{2}}{m_{1}}$ is the mass ratio.

If a binary satisfies these two criteria, the binary is marked as a HMXB. Since the two criteria are independently checked at every time step, which criterion is satisfied first is not important.

\subsection{Setup and Initial Conditions}

We model dark matter halo with virial temperatures $\sim 1000-2000 \mathrm{~K}$ at the redshift $20 \lesssim$ $z \lesssim 30$ as a birth place of Pop III formation. The initial positions of the stars are generated quasirandomly via a Monte Carlo realization with the assumption that they formed inside a shared Keplerian gas disk at the center of the host dark matter halo. Correspondingly, the initial velocities of the stars are assigned to be circular, parallel to the disk plane, and Keplerian at the instant the simulation begins.

In the simulations by [25], multiple protostars formed several AU apart from each other. The masses of the stars follow the initial mass function (IMF) with $\alpha=0.17\left(\frac{d N}{d M}=M^{-\alpha}\right.$, [26]), $M_{\max }=$ $140 \mathrm{M}_{\odot}$ and $M_{\min }=0.1 \mathrm{M}_{\odot}$. we run simulations with number densities $n_{6}$ and $n_{4}$. In addition to that, we explore several different configurations: cases with a star group of $N=5$ stars, a star group of $N=10$ stars, and collisions between two groups of $N=5$ stars. 


\begin{tabular}{ccc}
\hline & $n_{6}$ & $n_{4}$ \\
\hline runs & 86 & 86 \\
$\left\langle a_{t=500 \mathrm{yr}}\right\rangle[\mathrm{AU}]$ & 1.37 & 1.42 \\
$\tau_{\mathrm{df}}[\mathrm{yr}]$ & $\sim 10^{13}$ & $\sim 10^{15}$ \\
Companion stars & 3 rd massive star, $11 \sim 12 \mathrm{M}_{\odot}$ \\
$P_{\mathrm{HMXBc}}$ & 0.070 & 0.070 \\
$F_{\mathrm{HMXB}}\left[10^{-4} \mathrm{M}_{\odot}^{-1}\right]$ & 4.6 & 4.6 \\
\hline
\end{tabular}

Table 1: Summary of results for simulations of 5-body groups forming on scales of $\simeq 10$ AU. $\left\langle a_{t=5000 \mathrm{yr}}\right\rangle$ indicates the semi-major axis at $t=5000 \mathrm{yr}$, while $\tau_{\mathrm{df}}$ represents the dynamical friction timescale required for $a_{t=5000 \mathrm{yr}}$ to shrink to $a_{\mathrm{RL}}$. $P_{\mathrm{HMXBc}}$ is the fraction of runs in which a HMXBc forms, and $F_{\mathrm{HMXB}}$ is the number of HMXBc formed across all simulations, normalized by the total mass of the stars in the simulations.

\section{Results}

Here, we summarize the findings of our $N$-body simulations, focusing in particular on the properties of the most compact binaries found for each set of runs.

\subsection{5-body simulations}
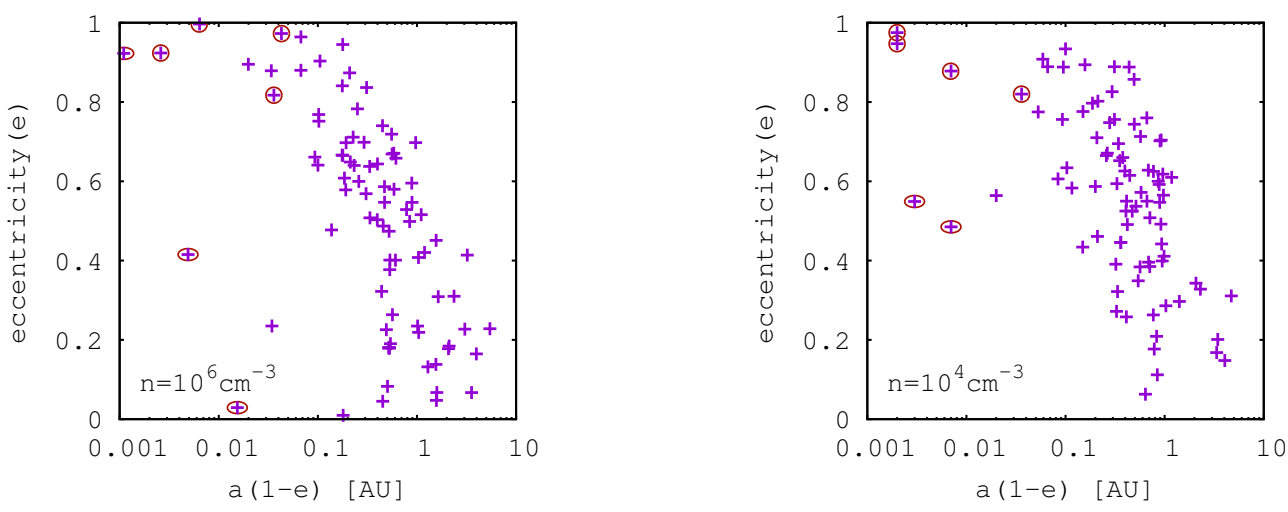

Figure 1: Left: Pericenter distance - eccentricity distribution plot for $n_{6}$. Two HMXB candidates (HMXBc) have been produced. Right: The same distribution plot for $n_{4}$. The circled points indicate HMXBc whose mass transfer via RLOF may occur periodically due to their eccentric orbits. The elliptically-circled point indicates an HMXBc whose semimajor axis is smaller than $a_{\mathrm{RL}}$, so the mass transfer will be steady. There are a couple of binaries with pericenter distance smaller than $R_{\mathrm{RL}}$, but they are excluded because $M_{2}<8 \mathrm{M}_{\odot}$.

We performed 86 runs of 5-body simulations for each of the number density values $n_{6}$ and $n_{4}$. We use the same initial conditions for each set of runs for proper comparisons.

In the $n_{6}$ calculations, the most common scenario is that $S_{1}$ always forms the most compact binary almost immediately, while stellar scatterings are most common during the first few years to about 40 years. Thereafter, a multiple system usually survives and stabilizes, while less massive stars are ejected. 
In our 86 runs, the last surviving binary is typically of type $\mathrm{B}_{13}$ (pairing of the most massive and third most massive stars). with total mass of $130 \mathrm{M}_{\odot}$ and $\left\langle M_{\mathrm{S}_{3}}\right\rangle=11 \mathrm{M}_{\odot} .\left\langle a_{t=5000 \mathrm{yr}}\right\rangle=$ $1.87 \mathrm{AU}$ and the minimum semi-major axis is $0.2 \mathrm{AU}$. The corresponding dynamical friction time scale is $\sim 10^{13} \mathrm{yr}$. The ejected stars have speeds of $(10-100) c_{\mathrm{s}}$, and $c_{\mathrm{s}} \sim 4 \mathrm{~km} / \mathrm{s}$. Six of the HMXB candidates (HMXBc hereafter) have been formed in all runs $\left(P_{\mathrm{HMXBc}}=0.070\right)$. For later use, let us define $F_{\mathrm{HMXB}}$ as the number of $\mathrm{HMXBc}$ formed across all simulations, normalized by the total mass of the stars. Then $F_{\mathrm{HMXBc}}=4.6 \times 10^{-4} \mathrm{M}_{\odot}^{-1}$ (This term will be used to estimate the $\mathrm{X}$-ray luminosity and is one of the primary results of our study).

The outcomes of the $n_{4}$ simulations are quite similar: a triple or higher multiple forms in 65 of 86 runs, and $\left\langle a_{t=5000 \mathrm{yr}}\right\rangle=1.42 \mathrm{AU}$. Furthermore, due to the same number of HMXBc, $P_{\mathrm{HMXBc}}$ and $F_{\mathrm{HMXBc}}$ are the same. One notable difference is that for $n_{4}$, the dynamical friction time scale is longer by 2 orders of magnitude compared to $n_{6}$, because this quantity is inversely proportional to the number density.

We present the distribution of eccentricity for pericenter distance in Figure 1. In particular, the left panel shows the distribution for $n_{6}$ calculations and the right panel for $n_{4}$. The circled point indicates $\mathrm{HMXBc}$ with distance at pericenter shorter than the corresponding Roche-Lobe radius.
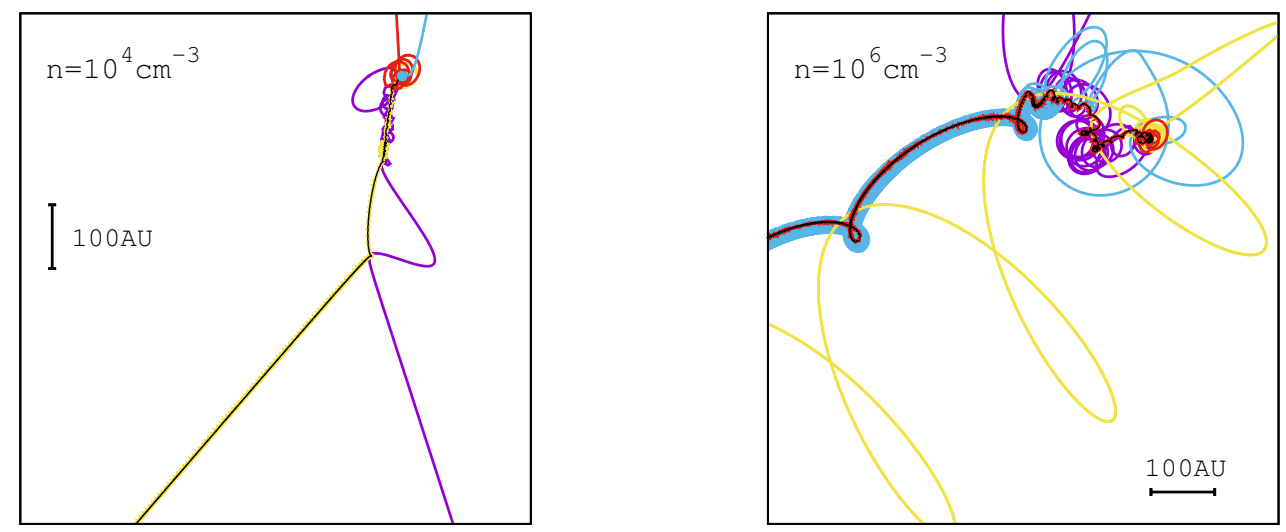

Figure 2: Trajectories up to $t=2000$ yr for 5-body simulations with identical initial conditions but different densities: $n_{4}$ (left panel) and $n_{6}$ (right panel). Despite having the same starting point, the trajectories of the 5 stars evolve quite distinctly in the two backgrounds due to the different magnitudes of dynamical friction. The two simulations for different bound systems: a binary for $n_{4}$ (black+yellow line) and a quartet for $n_{6}$ (black+red+blue+yellow lines).

Figure 2 shows two sample trajectories of 5 stars with low number density (left panel) and high number density (right panel) after $1000 \mathrm{yr}$. They were given identical initial conditions for the run, but their trajectories have developed differently. In Figure 3, at $800 \mathrm{yr}$, even though the same stars form a triple and the same star is ejected for both number densities, their trajectories are clearly different.

There is no noticeable difference in the overall results between the $n_{4}$ and $n_{6}$ runs-quantities such as $\left\langle a_{t=5000 \mathrm{yr}}\right\rangle$, the dynamical friction time scale $\tau_{\mathrm{df}}$, the total mass of the most compact binary (or which star forms the compact binary with $S_{1}$ ) as summarized in Table 1. For both number densities, the companion star of the binary is typically the third most massive star $\mathrm{S}_{3}$.

To sum up, we find that $P_{\mathrm{HMXBc}} \sim 7 \%$ of our simulations form $\mathrm{HMXBc}$, regardless of the 

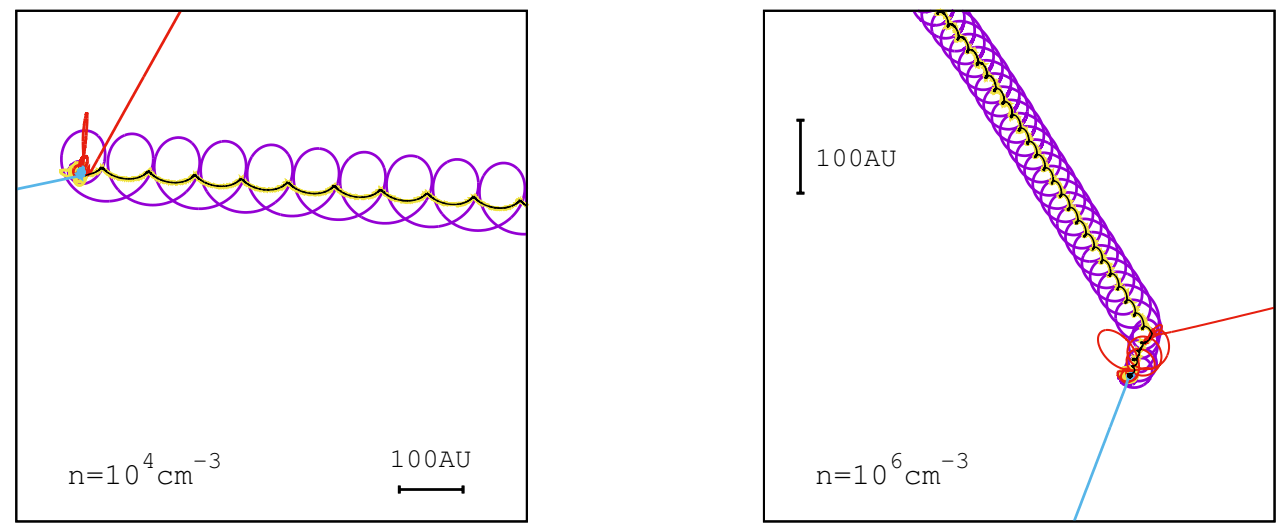

Figure 3: Trajectories of two 5-body simulations with identical initial conditions but different ambient gas densities. The simulations have formed triples with the identical stars (black+yellow+purple lines; contrast with 2) at $t=1000 \mathrm{yr}$, despite differences in the trajectories of each star and the center of mass.

\begin{tabular}{cccccc}
\hline Scenario & 1 & 2 & 3 & $4 \mathrm{~A}$ & $4 \mathrm{~B}$ \\
\hline runs & 54 & 30 & 30 & 30 & 30 \\
$\left\langle a_{t=500 \mathrm{yr}}\right\rangle[\mathrm{AU}]$ & 1.0 & 1.1 & 0.90 & 1.8 & 1.6 \\
$\left\langle a_{\mathrm{B}_{12}}\right\rangle[\mathrm{AU}]$ & 1.6 & 1.5 & 1.8 & 2.4 & 2.0 \\
$\left\langle a_{\mathrm{rest}}\right\rangle[\mathrm{AU}]$ & 0.72 & 0.15 & 0.42 & 1.2 & 0.23 \\
$P_{\mathrm{HMXBc}}$ & 0.33 & 0.33 & 0.27 & 0.13 & 0.27 \\
$F_{\mathrm{HMXBc}}\left[10^{-4} \mathrm{M}_{\odot}^{-1}\right]$ & 15 & 11 & 9.0 & 4.2 & 8.4 \\
\hline
\end{tabular}

Table 2: Summary of the results from 10-body simulations. We have considered five different scenarios. Scenario 1 simulated the 10-body version of the 5-body calculation, i.e. an isolated star group. The other scenarios all involve collisions of two 5-body star groups. Scenario 2 is a head-on collision of two coplanar, co-rotating star groups. Scenario 3 also collided two groups of stars with co-rotating orbital planes, but with an impact parameter comparable to the sizes of the groups, which results in an inspiral and eventual merger. Scenarios 4A and 4B are similar to scenario 3, except that the orbital planes of the colliding star groups had mutual inclinations of $45^{\circ}$ and $135^{\circ}$, respectively.

gas density value. Normalized to the total stellar mass in the simulations, the number of HMXBc formed per stellar mass is $F_{\mathrm{HMXB}} \approx 4.6 \times 10^{-4} \mathrm{M}_{\odot}^{-1}$.

\subsection{0-body simulations}

We now explore several different configurations for the star group, and run several sets of simulations with 10 stars (instead of 5) with $n=10^{6} \mathrm{~cm}^{-3}$. These are: (1) 10-body version of the calculation presented above; (2) head-on crash of two star groups containing 5 stars each; (3) a close encounter and subsequent inspiral and merger of two star groups containing 5 stars each.

We generate stellar masses in the same way as for the 5-body case, but with a larger value for the parameter $M_{\max }=300 \mathrm{M}_{\odot}$. We have run each simulation for $500 \mathrm{yr}$. The results of these simulations are summarized in Table 2. We briefly discuss each one, as follows. 


\subsubsection{Scenario 1: 10-body group in isolation}

We set up the simulations as in the 5-body calculations, but with 10 stars.

We find that $\left\langle a_{t=500 \mathrm{yr}}\right\rangle=1.0 \mathrm{AU}$. Interestingly, there are 18 out of 54 cases in which $a<a_{\mathrm{RL}}$. There is a large difference in scale between $a_{\mathrm{B}_{12}}$ and $a_{\text {rest }}$, where $a_{\mathrm{B}_{12}}$ is the semi-major axis of $\mathrm{B}_{12}$ (binary made up of the two most massive stars) and $a_{\text {rest }}$ is the semi-major axis of the binary stars other than $S_{1}$ and $S_{2}$. This is a common feature of the 10-body simulations: they often end up with triples whose inner binary is $B_{\text {rest }}$ while the outer binary is $B_{12}$. Our simulations yield $\left\langle a_{\text {rest }}\right\rangle=0.72 \mathrm{AU}$ and $\left\langle a_{\mathrm{B}_{12}}\right\rangle=1.6 \mathrm{AU}$. Also note that, in 14 out of $18 \mathrm{HMXBc}$, the binary is $\mathrm{B}_{\text {rest }}$ (i.e. it is not made up of the two most massive stars).

We find that a HMXBc forms in a larger fraction of these simulations than in the 5-body case, $P_{\mathrm{HMXBc}}=0.33$, for the obvious reason that there are more stars. Per unit stellar mass in the simulations, the number of HMXB candidates is $F_{\mathrm{HMXB}}=1.5 \times 10^{-3} \mathrm{M}_{\odot}^{-1}$, which is a factor $\approx 3$ higher than we found for the 5-body case.

\subsubsection{Scenario 2: Collision between two 5-star groups - head-on collision}

In scenario 2, two groups of 5 stars collide head-on. The initial conditions for two groups of 5 stars are generated randomly in the same way as for 5-body simulations. Their separations are two to three times their sizes and the mutual inclination is zero. The initial relative speed of the groups is roughly the speed of sound and the center of mass of one group is set to move directly toward the center of mass of the other group.

We find that prior to colliding, each group forms a compact binary of type $\mathrm{B}_{12}$ (the most and second-most massive star). When the two groups collide, those two binaries that existed before the collision were broken and the two most massive stars of each group form a new compact binary with high chances. The average $\left\langle a_{t=500 \text { yr }}\right\rangle$ is $1.1 \mathrm{AU}$, but $\left\langle a_{\mathrm{B}_{12}}\right\rangle=1.5 \mathrm{AU}$ and $\left\langle a_{\text {rest }}\right\rangle=0.15 \mathrm{AU}$, meaning that the most compact binaries are not formed from the most massive stars. The shorter average separations may be a result of a larger number of early 3-body scatterings, which act to harden the group as a whole.

$\mathrm{HMXBc}$ form in 10 out of 30 runs, and they are not of type $\mathrm{B}_{12}$. However, we find a rate of HMXB formation per stellar mass $F_{\mathrm{HMXB}}=1.1 \times 10^{-3} \mathrm{M}_{\odot}^{-1}$; this is higher than in the 5-body case and comparable to Scenario 1 above. Sample trajectories for one of the simulations of scenario 2 are depicted in Figure 4.

\subsubsection{Scenario 3: Collision between two 5-star groups - spirally merging case}

We have used the same orbital parameters for the two groups as in Scenario 2, except that we now set the impact parameter to be of order the size of the group, whereas it was set to zero in Scenario 2. So they merge with a spiral motion.

We find that $\left\langle a_{t=500 \mathrm{yr}}\right\rangle=0.90 \mathrm{AU},\left\langle a_{\mathrm{B}_{12}}\right\rangle=1.8 \mathrm{AU}$ and $\left\langle a_{\text {rest }}\right\rangle=0.42 \mathrm{AU}$. The average separation lies between what we find in Scenarios 1 and 2. This can be interpreted as being due to the fact that these simulations (in which the two groups merge gradually via inspiral) have more close 3-body interactions than in Scenario 1 (in which 10 stars in quasi-Keplerian orbits evolve in isolation) but fewer such interactions than in Scenario 2 (in which the two groups merge head-on).

HMXBs form in 8 out of 30 runs and, as with Scenarios 1 and 2, none of the HMXBs are made up of the two most massive stars. We find a similar HMXB formation rate per stellar mass, 

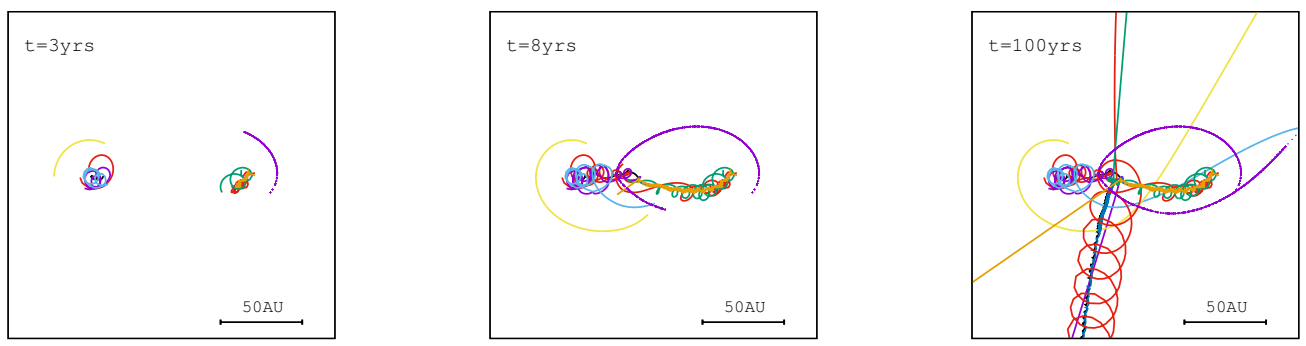

Figure 4: 10-body head-collision (scenario 2). In these sample trajectories, five stars in each group are in nearly Keplerian motion. The groups move towards each other with a relative velocity of $\sim c_{\mathrm{S}}$ (left panel). At $t \approx 8 \mathrm{yr}$ (middle panel), the two haloes begin to merge. During the merger, all ten stars undergo stellar scatterings (right panel). One triple (black+dark blue+red lines) has been formed and it is moving in -y direction. In these head-on collisions, it is likely for multiple systems that existed before merging to be broken, while a few new multiple systems are formed.
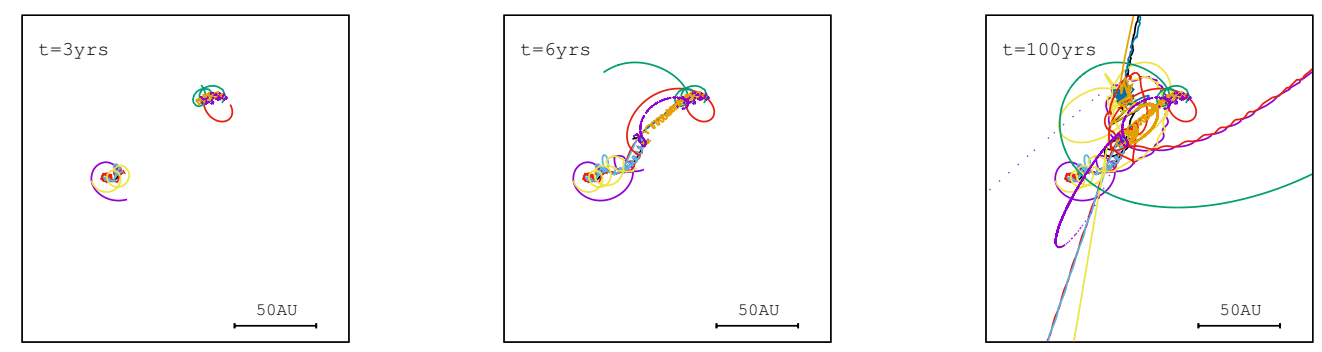

Figure 5: 10-body spirally merging case (scenario 3). As with scenario 2, two groups of 5 stars are set on a collision cours. However, in this scenario the impact parameter is $\sim 2-3$ times the size of groups, whereas in scenario 2 it is set to be zero. The groups are approaching each other at the relative velocity of $c_{\mathrm{s}}$ (left panel). At $t=8 \mathrm{yr}$, the groups are about to merge (middle panel). After some time (right panel), two binaries are ejected (red and purple lines in the $x$-direction and black and blue lines in the $y$-direction). In this sample case, the most compact binary is the one ejected in the y direction (black+blue lines) with $a=0.8 \mathrm{AU}$ at $t=100 \mathrm{yr}$. A difference between this scenario and the head-on collision is that compact systems are more likely to survive the merger.

$F_{\mathrm{HMXB}}=9.0 \times 10^{-4} \mathrm{M}_{\odot}^{-1}$. Sample trajectories from one of the simulations for Scenario 3 are shown in Figure 5.

\subsubsection{Scenarios 4A and 4B: Collision between two 5-body groups - spirally merging case with inclinations of $\mathbf{4 5}$ degrees and 135 degrees}

In these two scenarios, the difference compared with those previous scenarios is that the orbital plane of one star group is tilted, or non-zero mutual inclination $i$. We take the inclination at $i=45^{\circ}$ (nearly co-rotating) for scenario $4 \mathrm{~A}$, and $i=135^{\circ}$ (nearly counter-rotating) for scenario $4 \mathrm{~B}$. The groups are initially placed at a separation of two to three times their sizes, and set in motion at the same speeds as for the inspiral case (scenario 3 ).

We find that $\left\langle a_{t=500 \mathrm{yr}}\right\rangle=1.8 \mathrm{AU},\left\langle a_{\mathrm{B}_{12}}\right\rangle=2.4 \mathrm{AU}$, and $\left\langle a_{\text {rest }}\right\rangle=1.2 \mathrm{AU}$ for scenario $4 \mathrm{~A}$. In scenario $4 \mathrm{~B}$, we find $\left\langle a_{t=500 \mathrm{yr}}\right\rangle=1.6 \mathrm{AU},\left\langle a_{\mathrm{B}_{12}}\right\rangle=2.0 \mathrm{AU}$, and $\left\langle a_{\text {rest }}\right\rangle=0.23 \mathrm{AU}$. In $70 \%$ of the runs for both scenarios, the two most massive stars form the most compact binary. Stellar binaries end up with somewhat closer separations in the nearly counter-rotating case, due to the fact that the 

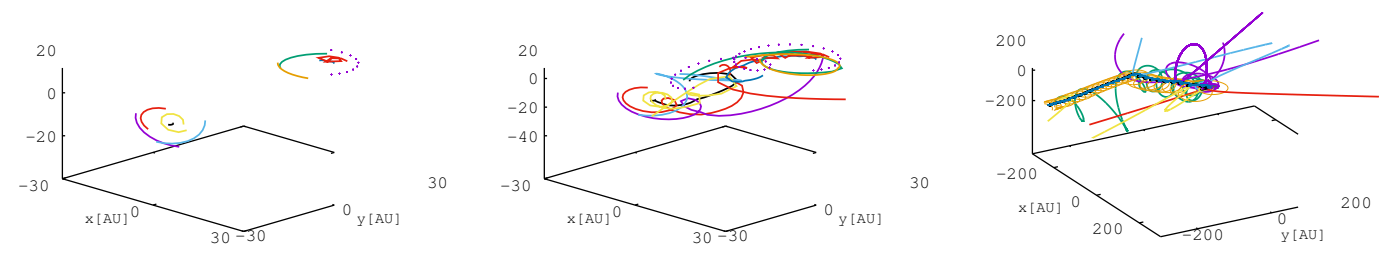

Figure 6: Trajectories for scenario 4A, a collision between two 5-body groups, each on quasi-Keplerian orbits, but with the orbital plane of the two groups tilted with respect to each other at an inclination $i=45^{\circ}$. The setup is the same as scenario 3, except for the mutual inclination of the orbital planes of the colliding star groups. The left panel shows the two groups on a collision course. At $t=4 \mathrm{yr}$, the halos are about to merge (middle panel). After $t=10 \mathrm{yr}$ (right panel), a triple having the most compact binary are ejected [black and blue lines (inner binary) and brown line].
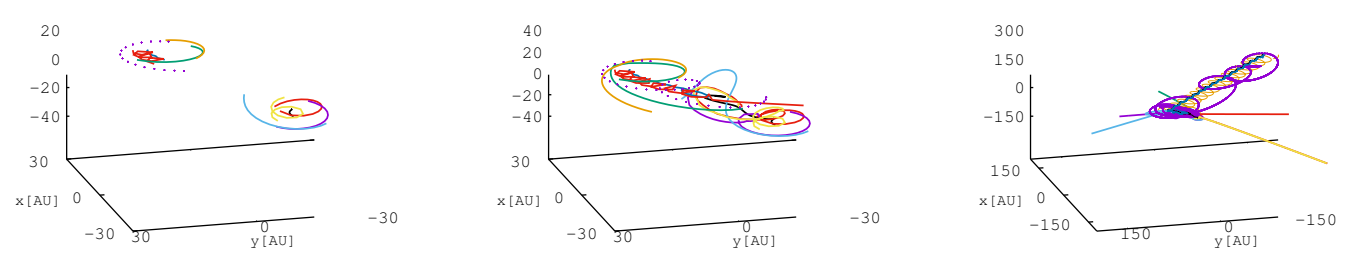

Figure 7: Trajectories for scenario 4B-the same as scenario 4A (Figure 6), but with the two groups nearly counter-rotating with respect to each other $\left(i=135^{\circ}\right)$. At $t=4 \mathrm{yr}$, the groups are about to merge (middle panel) and after $t=10 \mathrm{yr}$ (right panel), a quartet containing the most compact binary is ejected toward the upper right of the panel [black+blue lines (inner binary), brown and thick purple lines].

net angular momentum of the merged star group is smaller. Indeed, we find a total of four HMXBc across all the $i=45^{\circ}$ simulations, and eight in a same number of $i=135^{\circ}$ simulations.

For the same reason, we find a larger fraction of HMXB candidates per stellar mass simulated in the nearly counter-rotating case $\left(F_{\mathrm{HMXB}}=8.4 \times 10^{-4} \mathrm{M}_{\odot}\right)$ compared to the nearly co-rotating case $\left(F_{\mathrm{HMXB}}=4.2 \times 10^{-4} \mathrm{M}_{\odot}\right)$. The overall formation rate of HMXB candidates is lower for both cases than the cases in which all the stellar orbits were nearly coplanar (Scenarios 1, 2 and 3), plausibly due to the additional degree of freedom in the stellar orbits. Still, the value of $F_{\mathrm{HMXB}}$ is within a factor of a few for all of our simulations.

Sample trajectories from runs for scenarios 4A and 4B are depicted in Figure 6 and Figure 7, respectively.

\section{Discussion}

\subsection{Binary evolution and formation of HMXB candidates}

Our simulations show that if protostellar clouds fragment and form stars in close groups on scales of $\gtrsim 10 \mathrm{AU}$, as in [6], then a small fraction of groups can form HMXBs. We briefly discuss the dynamics of HMXB formation in our simulations, then move on to discuss the astrophysical implications of our findings. 
According to our simulations, scatterings play a major role in making a compact binary. The background potential and the dynamical friction play a secondary role, by allowing the most compact binary (or triple) to remain near the center of mass of the halo and for other stars to return and scatter again and again.

We find that on average, the number of HMXB candidates formed per stellar mass, $F_{\mathrm{HMXB}}$, depends on the number and orientation of close 3-body encounters. Our results indicate that if fewer number of stars are ejected during scattering and their interactions are coplanar, $F_{\mathrm{HMXB}}$ may be somewhat higher. It is important to note that the value of $F_{\mathrm{HMXB}}$ does not vary by more than a factor $\approx 3$ throughout all scenarios. We interpret this lack of a significant variation in $F_{\mathrm{HMXB}}$, for such a diverse set of initial conditions and ambient gas densities, to mean that our values are not far from the one that results from similar stellar encounters in nature.

\subsection{The effect of migration on the formation of HMXBs}

The migration process could be one another way to harden the stellar group through a gaseous disk. The migration could occur as the protostars form-[25] found significant accretion from the protostellar disk onto the most massive protostar, and did not follow the evolution of the system beyond this stage.

We evaluate the possible role of disk migration on the separation of Pop III stars by considering a steady, geometrically thin disk with an $\alpha$ viscosity ([32], [33]). We adopt a disk with $\alpha=0.01$ and an accretion rate $\dot{m} \sim 10^{-3} \mathrm{M}_{\odot} \mathrm{yr}^{-1}$ ([34], [35]), who considered the structure of accretion disks around Pop III stars at high redshifts.

We estimate the migration timescale $\tau_{\mathrm{mig}}$ ([36]) for a representative binary system with primary mass $M_{1}=120 \mathrm{M}_{\odot}$ and secondary mass $M_{2}=11 \mathrm{M}_{\odot}$, based on the mean values found across our simulations.

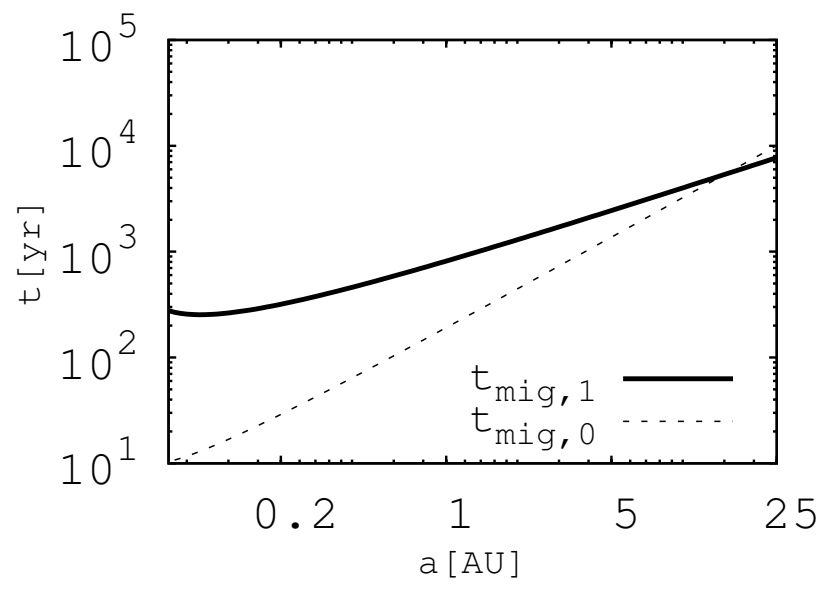

Figure 8: The migration timescales for a circumbinary protostellar disk, based on the typical binary properties of our simulations. The unperturbed Type II migration timescale, $\tau_{\mathrm{mig}, 0}$ (dashed line) shows the timescale assuming that the secondary mass is much smaller than the local disk mass. A longer timescale $\tau_{\mathrm{mig}, 1}$ (solid line) is expected if the secondary mass is large compared to the disk mass.

We plot the two migration timescales $\tau_{\mathrm{mig}, 0}$ and $\tau_{\mathrm{mig}, 1}$ in Figure 8 . The unperturbed Type II 
migration timescale, $\tau_{\text {mig, } 0}$ (dashed line) shows the timescale assuming that the secondary mass is much smaller than the local disk mass. A longer timescale $\tau_{\mathrm{mig}, 1}$ (solid line) is expected if the secondary mass is large compared to the disk mass. Both of these timescales are shorter than both the typical lifetimes of protostellar disks, as well as the lifes of the stars themselves ${ }^{1}$. This suggests, along with findings that the disks may be able to survive even under radiation feedback ([37], [38]), that disk migration could lead to initial stellar separations smaller than what we have assumed, making the formation of HMXBs via stellar scatterings more favorable.

We submit that the HMXB formation rates inferred from our simulations be taken as a conservative estimate, with possible additional contributions from channels other than stellar scattering.

\subsection{X-ray output}

As discussed in $\S 1, \mathrm{HMXBs}$ are believed to be a major source of X-rays in the early universe. Observations of nearby star-forming galaxies suggest that their X-ray luminosities (which are dominated by HMXBs) scale linearly with their star formation rate ([39], [40], [41], [42]). In the linear regime, the X-ray luminosity of the local universe is given by [43],

$$
L_{2-10 \mathrm{keV}}^{\text {local }}=3 \times 10^{39} \times \frac{\mathrm{SFR}}{\mathrm{M}_{\odot} \mathrm{yr}^{-1}}\left[\mathrm{erg} \mathrm{s}^{-1}\right]
$$

where SFR is the star formation rate.

In the following, based on the results of our simulations, we are going to quantitatively evaluate the relation between X-ray luminosity and SFR, and compare it with equation (4.1). We can write the X-ray luminosity as

$$
\begin{aligned}
L_{2-10 \mathrm{keV}}= & L_{\mathrm{Edd}} \times f_{\mathrm{Edd}} \times f_{2-10 \mathrm{keV}} \\
& \times t_{\mathrm{acc}} \times f_{\text {sur }} \times f_{\mathrm{esc}} \times F_{\mathrm{HMXB}} \times \mathrm{SFR} .
\end{aligned}
$$

Below, we discuss each quantity in equation (4.2).

1. $L_{\mathrm{Edd}}$, the Eddington luminosity, which scales with the typical mass of the $\mathrm{CO}$ engine $M_{\mathrm{CO}}$ as $1.3 \times 10^{38}\left(M_{\mathrm{CO}} / \mathrm{M}_{\odot}\right) \mathrm{erg} \mathrm{s}^{-1}$. Note that, for stars which leave behind a $\mathrm{BH}$ remnant $\left(M \gtrsim 25 M_{\odot}\right)$, we use the stellar mass as a proxy for the BH mass for simplicity, due to the theoretical uncertainties in evaluating the mass loss due to winds and during the transition to a BH. For stars with $M \lesssim 25 M_{\odot}$, we consider two limiting cases: one in which all the stars form BHs, and one in which all the stars form NSs, for which we assume a typical mass value of $1.4 \mathrm{M}_{\odot}$. However, this uncertainty plays a very small role for our results due to the fact that the number of HMXBs with a star of $M<25 \mathrm{M}_{\odot}$ is a small fraction of the total.

2. $f_{\text {Edd }}$, the typical ratio of the total radiative power emitted by HMXBs (the bolometric luminosity) to $L_{\text {Edd }}$. If the typical luminosity of a HMXB during an active phase is Eddington, then $f_{\text {Edd }}$ is effectively the mean duty cycle. Other studies have adopted values ranging from 0.1 to 0.5 ([42], [44], [45]). we take as our fiducial value $f_{\text {Edd }}=0.1$.

\footnotetext{
${ }^{1}$ Note that, if the adopted value of $\alpha$ were higher, e.g. $\sim 0.1-1$ as suggested by recent simulations of Pop III protostellar disks [60], the migration timescale would become even shorter.
} 
3. $f_{2-10 \mathrm{keV}}$, the fraction of the bolometric luminosity that is emitted between 2 and $10 \mathrm{keV}$. Observational estimates vary between 0.1 and 0.8 ([46], [47]).

4. $t_{\mathrm{acc}}$, the time that a massive binary spends as a HMXB, with the less massive star donating mass to the $\mathrm{CO}$ companion. If the two stars form simultaneously and form a compact binary before the more massive member dies to become a $\mathrm{CO}$, then this is simply $t_{2}-t_{1}$, the difference in the lifetimes of the stars.

5. $f_{\text {sur }}$, the fraction of HMXB candidates identified in our simulations that actually survive to become HMXBs. This quantity accounts for possible disruptions of binaries, due to (a) the merger of the stars during main sequence and post-main sequence evolution ([48]); (b) the more massive star getting kicked following a supernova explosion ([49], [50]); and (c) subsequent disruptions by stellar scatterings that were not captured by our simulations.

6. $f_{\text {esc }}$, the fraction of X-rays that escape the galaxy. Unless the environment of the HMXBs are Compton-thick, which is unlikely for the low-mass galaxies of interest, we expect $f_{\text {esc }} \lesssim 1$.

7. $F_{\mathrm{HMXB}}$, the number of HMXBs formed per stellar mass. This is the main output of our simulations. ${ }^{2}$ Whereas previous theoretical works had arrived at this value by extrapolating the locally observed value with an assumed redshift evolution, or with free parameters, here we provide an estimate based on suites of $N$-body simulations whose initial conditions are motivated by cosmological simulations of Pop III star formation. A higher mass fraction in BHs should result in more X-ray production per unit star formation, and a more pronounced effect on the IGM kinetic temperature and greater thermal feedback on early galaxy formation and evolution ([17], [18]).

In all of our simulations independent of the number of stars in the group and collision configurations, we find $F_{\mathrm{HMXB}} \sim 10^{-3}$, varying by less than a factor of 4 between the lowest and the highest values (see Table 3).

Finally, we can write $L_{2-10 \mathrm{keV}}$ as follows :

$$
\begin{aligned}
\frac{L_{2-10 \mathrm{keV}}}{\mathrm{SFR}}=0.33 & \times \frac{M_{\mathrm{BH}}}{\mathrm{M}_{\odot}} \times \frac{f_{\mathrm{Edd}}}{0.1} \times \frac{f_{2-10 \mathrm{keV}}}{0.1} \times \frac{t_{\mathrm{acc}}}{\mathrm{Myr}} \\
& \times \frac{f_{\text {sur }}}{0.5} \times \frac{f_{\mathrm{esc}}}{0.5} \times \frac{F_{\mathrm{HMXB}}}{10^{-3} \mathrm{M}_{\odot}^{-1}} \\
& \times \frac{10^{39} \mathrm{erg} \mathrm{s}^{-1}}{\mathrm{M}_{\odot} \mathrm{yr}^{-1}} .
\end{aligned}
$$

Based on our choices of the factors, $f_{\mathrm{Edd}}=0.1, f_{2-10 \mathrm{keV}}=0.1, f_{\mathrm{sur}}=0.5$ and $f_{\mathrm{esc}}=0.5$, we can estimate the normalized X-ray luminosities per SFR, $L_{2-10 \mathrm{keV}} / \mathrm{SFR}$. We report this quantity for each of our models in Table 3. It varies from a minimum of 37 to a maximum of 450 among the studied scenarios.

\footnotetext{
${ }^{2}$ Note that $F_{\mathrm{HMXB}}$ has units $\mathrm{M}_{\odot}^{-1}$; we use the capital letter to distinguish it from the dimensionless fractions represented by $f$.
} 


\begin{tabular}{|c|c|c|c|c|c|c|c|}
\hline & 5-body $\left(n_{6}\right)$ & 5-body $\left(n_{4}\right)$ & Sce. 1 & Sce. 2 & Sce. 3 & Sce. $4 A$ & Sce. $4 B$ \\
\hline$M_{1}\left[\mathrm{M}_{\odot}\right]\left(\tau_{\text {life }, 1}[\mathrm{Myr}]\right)$ & $110(2.5)$ & $88(2.9)$ & $110(2.5)$ & $120(2.4)$ & $110(2.4)$ & $160(2.0)$ & $110(2.4)$ \\
\hline$M_{2}\left[\mathrm{M}_{\odot}\right]\left(\tau_{\mathrm{life}, 2}[\mathrm{Myr}]\right)$ & $12(17)$ & $11(18)$ & $18(11)$ & $45(4.8)$ & $28(7.1)$ & $63(3.7)$ & $63(3.7)$ \\
\hline$t_{\mathrm{acc}}\left(=\tau_{\mathrm{life}, 2}-\tau_{\mathrm{life}, 1}\right)[\mathrm{Myr}]$ & 14 & 15 & 8.6 & 2.5 & 4.7 & 1.7 & 1.3 \\
\hline$F_{\mathrm{HMXB}}\left[10^{-4} \mathrm{M}_{\odot}^{-1}\right]$ & 4.6 & 4.6 & 15 & 11 & 9.0 & 4.2 & 8.4 \\
\hline$L_{2-10 \mathrm{keV}} / \mathrm{SFR}\left[10^{39} \mathrm{ergs}^{-1} \mathrm{M}_{\odot}^{-1} \mathrm{yr}\right]$ & 220 & 200 & 450 & 110 & 160 & 37 & 40 \\
\hline$L_{2-10 \mathrm{keV}} / L_{2-10 \mathrm{keV}}^{\text {local }}$ & 75 & 67 & 150 & 36 & 52 & 12 & 13 \\
\hline$\frac{\eta_{\mathrm{GRB}, \text { PopIII }}}{\eta_{\mathrm{GRB}, \text { PopI } / \mathrm{II}}}$ & 2.2 & 2.2 & 7.0 & 5.4 & 4.3 & 2.0 & 4.0 \\
\hline
\end{tabular}

Table 3: Summary of the results of X-ray luminosity and GRB efficiencies. In the table, second and third columns correspond to 5-body calculations and the rest columns (from Sce. 1 to Sce. 4B) are the results from 10-body calculations with the number density of $10^{6} \mathrm{~cm}^{-3}$. According to the ratio $L_{2-10 \mathrm{keV}} / L_{2-10 \mathrm{keV}}^{\text {local }}$, where $L_{2-10}^{\text {local }} \mathrm{keV} / \mathrm{SFR}=3 \times 10^{39} \mathrm{erg} \mathrm{s}^{-1}$, it is expected that the $2-10 \mathrm{keV} \mathrm{X}$-ray luminosity is $\sim 10^{2}$ times larger than the X-ray luminosity of the local universe. These larger values essentially result from the large mass of the star 1. In the bottom row, we have listed the LGRB efficiency, derived from each set of simulations. The higher HMXB formation rates, lead to larger efficiencies than the estimated value for Pop I/II stars, $\eta_{\mathrm{GRB}, \mathrm{PopI} / \mathrm{II}} \simeq 4.2 \times 10^{-6}$ with beaming factor of $1 / 50$ ([53]).

These $L_{\mathrm{X}}$-to-SFR ratios are $\sim 40-150$ higher than what is observed in the local Universe. This result is qualitatively consistent with the findings of [61] and [62], who find an increase in the $L_{X}$-to-SFR ratios toward $z \gtrsim 4$. Our high $L_{X}$-to-SFR values stem from the large mass of the $\mathrm{HMXBc}$ primary, and the relatively low mass of the secondary. The former leads to a higher Eddington luminosity compared to typical stellar-mass BHs $\left(\sim 3 \mathrm{M}_{\odot}\right)$ in the local Universe, and the latter results in long stellar lifetimes, which in turn leads to longer $t_{\mathrm{acc}}$.

\subsection{Implications for the thermal history of the IGM and the $21 \mathrm{~cm}$ radiation}

A higher $L_{X}$-to-SFR ratio implies that IGM heating will occur earlier than commonly thought. The thermal history of the IGM can be probed in the $21 \mathrm{~cm}$ line, which is observable in absorption (or in emission), depending on whether the spin temperature of the IGM is below (or above) the CMB temperature.

If the IGM heats early, as suggested by our estimates of the X-ray emission of early galaxies, the $21 \mathrm{~cm}$ absorption line appears earlier, and the "dip" as a function of redshift caused by adiabatic cooling is not as deep and not as sharp as in the case of late heating as it would otherwise (see Figure 2 in [63]). Another consequence of early, intense heating is that the temperature of the IGM could become high enough, to suppress the formation of low-mass galaxies ([17]) and the growth of their nuclear BHs ([18]).

\subsection{Implications for Gamma Ray Bursts from Pop III stars}

As discussed in $\S 1$, the fraction of HMXBs at high redshifts has potential implications for the expected rates of LGRBs from Pop III stars. Binary systems more easily satisfy the collapsar model ([51]) with respect to single stars ([52]). Binary stars can spin up the helium core of the progenitor star via tidal coupling and spin-orbit locking. Further, RLOF can strip the hydrogen envelope during a common-envelope phase without reducing the rotation of the helium core [53]. This is especially important for Pop III stars, whose heavier hydrogen envelopes would be more 
difficult to shed in isolation. Therefore, compact binary systems, or HMXBs, constitute a promising channel to produce LGRBs from Pop III stars.

We can use our results for the formation rates of Pop III HMXBs to estimate the fraction of LGRBs from Pop III stars. [53] quantified the GRB formation efficiency as

$$
\eta_{\mathrm{GRB}} \simeq \eta_{\mathrm{BH}} \eta_{\text {bin }} \eta_{\text {close }} \eta_{\text {beaming }}
$$

where $\eta_{\mathrm{BH}}$ is the number of $\mathrm{BH}$-forming stars resulting from a given total stellar mass, $\eta_{\text {bin }}$ is the binary fraction and $\eta_{\text {close }}$ is the fraction of sufficiently close binaries to undergo RLOF. For Pop I/II stars they calculated $\eta_{\mathrm{BH}} \simeq 1 /\left(700 \mathrm{M}_{\odot}\right)$. Combining this value with adopted values for the other parameters $-\eta_{\text {bin }} \sim 0.5, \eta_{\text {close }} \sim 0.3$, and $\eta_{\text {beaming }} \simeq(1 / 50)-(1 / 500)$ —yields $\eta_{\text {GRB }, \text { PopI } / I I} \sim$ $4.2 \times\left(10^{-6}-10^{-7}\right) \mathrm{M}_{\odot}^{-1}$.

Our work is a first attempt to fill the gap between simplified guesses stemming from the absence of detailed calculations for the fraction of close binaries and our theoretical knowledge. Starting with $F_{\mathrm{HMXB}}=\eta_{\mathrm{BH}} \eta_{\text {bin }} \eta_{\text {close }}$ and using the same value of $\eta_{\text {beaming }} \simeq(1 / 50)-(1 / 500)$ ([53]), we then infer $\eta_{\text {GRB,Pop III }} \simeq 4.8 \times\left(10^{-6} \sim 10^{-7}\right) \mathrm{M}_{\odot}^{-1}$ for the interacting 5-star case, and $2.2 \times\left(10^{-5} \sim 10^{-6}\right) \mathrm{M}_{\odot}^{-1}$ for the (most favorable) 10-body scenario.

Therefore, our results suggest that LGRB rates from Pop III stars could be comparable to or somewhat higher than the rates from Pop I/II stars. However, even with these rates, the probability of detecting a GRB from a Pop III star remains very low and rather uncertain. The probability depends on the mass function of Pop III stars, the likelihood that a Pop III star produces a GRB, the luminosity function of these bursts, and how efficiently such high-redshift GRBs can be identified. Accordingly, there is a wide range of published theoretical results based on different model assumptions ([56], [53], [57], [58]). In general, a detection of a GRB from a Pop III star will likely require a long time baseline, provided by a combination of the current Swift satellite and proposed future missions such as SVOM ([54]) and EXIST ([55]).

\section{Summary}

In this study, we used $N$-body simulations of the first stars to explore the formation, evolution, disruption and energy output of Pop III HMXBs. The code considers gravitational scattering of stars, dynamical friction, and the gravitational potential of ambient gas.

The initial conditions for the simulations (i.e. IMF, typical star separation in the host haloes, ambient densities) are taken from two different sets of cosmological simulations of Pop III formation, namely by [26] and [25]. We investigated star evolution in two backgound gas densities, a high-density case $\left(10^{6} \mathrm{~cm}^{-3}\right)$, and a lower-density one $\left(10^{4} \mathrm{~cm}^{-3}\right)$.

Based on the handful of protostars per halo that are found in the works quoted above, we simulated systems with 5 stars and systems with 10 stars. We found:

1. 5-body simulations: If stars form in compact groups separated by $\sim 10 \mathrm{AU}$, as is expected from turbulent fragmentation, stellar scatterings lead to a significant HMXB formation rate. In particular, we found that HMXBs form at a rate of a few per $10^{4} \mathrm{M}_{\odot}$ of stars formed, independent of the ambient gas density. 
2. 10-body simulations: We simulated 10 stars on separations of $\sim 10 \mathrm{AU}$, and evolved them as isolated quasi-Keplerian disks, or as two colliding groups with 5 stars each. For the latter, we ran several different sets of collision geometries. We found that the HMXB formation rate was a factor $\sim 1-3$ times higher than for the 5-body simulations, mainly due to the fact that the larger number of stars allowed for more hardening via stellar scattering.

All of the simulations suggest an X-ray luminosity per unit star formation that is a factor $\sim 10^{2}$ higher than what is observed in the local Universe (under the assumption that other variables such as the X-ray escape fraction from galaxies and the duty cycle of HMXBs do not differ significantly). These results are mostly due to the large mass of the most massive star of the HMXBc compared to that of the companion star, implying both a large $t_{\text {acc }}$ as well as a higher luminosity of the remnant $B H$. The fact that we found little variation in this quantity across all of our simulations suggests that this is a robust estimate. Additional factors, such as in-disk migration of nascent stars, could further increase the HMXB formation efficiency.

A direct consequence is that X-rays can heat the IGM rapidly at Cosmic Dawn. Signals of early heating can be probed via the $21 \mathrm{~cm}$ line radiation. In addition to the implications for the thermal history of the IGM, these high formation rates of HMXBs per stellar mass imply a higher GRB formation efficiency from Pop III stars in binaries. These predictions may be tested with a long baseline of observational data from $\underline{\mathrm{Swift}}$ in combination with future missions such as $\underline{\mathrm{SVOM}}$, EXIST.

\section{References}

[1] G. Hinshaw et al., Nine-year Wilkinson Microwave Anisotropy Probe (WMAP) Observations: Cosmological Parameter Results, ApJ Supp. 208 (2013) 19 [hep-th/1212 . 5226].

[2] Planck Collaboration et al., Planck 2015 results. XIII. Cosmological parameters, (2015) hep-th/1502.01589.

[3] T. Abel, G. L. Bryan, M. L. Norman, The Formation of the First Star in the Universe, Science 295 (2002) 93 [hep-th/0112088].

[4] M. J. Turk, T. Abel, B. O'Shea, The Formation of Population III Binaries from Cosmological Initial Conditions, Science 325 (2009) 601 [hep-th/ 0907 . 2919].

[5] A. Stacy, T. H. Greif, V. Bromm, The first stars: formation of binaries and small multiple systems, MNRAS 403 (2010) 45 [hep-th $/ 0908$. 0712].

[6] T. H. Greif, V. Springel, S. D. M. White, S. C. O. Glover, P. C. Clark, R. J. Smith , R. S. Klessen, V. Bromm, Simulations on a Moving Mesh: The Clustered Formation of Population III Protostars, ApJ 737 (2011) 75 [hep-th/1101.5491].

[7] V. Bromm, N. Yoshida, The First Galaxies, Ann. Rev. A\&A 49 (2011) 373 [hep-th/1102 . 4638].

[8] V. Springel, T. Di Matteo, L. Hernquist, Modelling feedback from stars and black holes in galaxy mergers, MNRAS 361 (2005) 776 [hep-th/ 0411108 ].

[9] G. Stinson, A. Seth, N. Katz, J. Wadsley, F. Governato, T. Quinn, Star formation and feedback in smoothed particle hydrodynamic simulations - I. Isolated galaxies, MNRAS 373 (2006) 1074 [hep-th/0602350]. 
[10] D. Sijacki, V. Springel, T. Di Matteo, L. Hernquist, A unified model for AGN feedback in cosmological simulations of structure formation, MNRAS 380 (2007) 877 [hep-th/ 0705 . 2238].

[11] A. Venkatesan, M. L. Giroux, J. M. Shull, Heating and Ionization of the Intergalactic Medium by an Early X-Ray Background, ApJ 563 (2001) 1 [hep-th/0108168].

[12] M. E. Machacek, G. L. Bryan, T. Abel, Effects of a soft X-ray background on structure formation at high redshift, MNRAS 338 (2003) 273 [hep-th / 0209326 ].

[13] S. P. Oh, Reionization by Hard Photons. I. X-Rays from the First Star Clusters, ApJ 553 (2001) 499 [hep-th/0005262].

[14] A. Venkatesan, J. Tumlinson, J. M. Shull, Evolving Spectra of Population III Stars: Consequences for Cosmological Reionization, ApJ 584 (2003) 621 [hep-th/ 0206390 ].

[15] M. Ricotti \& J. P. Ostriker, X-ray pre-ionization powered by accretion on the first black holes - I. A model for the WMAP polarization measurement, MNRAS 352 (2004) 547 [hep-th/ 0311003 ].

[16] J. R. Pritchard, S. R. Furlanetto, 21 -cm fluctuations from inhomogeneous X-ray heating before reionization, MNRAS 376 (2007) 1680 [hep-th/ 0607234 ].

[17] E. Ripamonti, M. Mapelli, S. Zaroubi, Radiation from early black holes - I. Effects on the neutral intergalactic medium, MNRAS 387 (2008) 158 [hep-th/ 0802 . 1857].

[18] T. Tanaka, R. Perna, Z. Haiman, X-ray emission from high-redshift miniquasars: self-regulating the population of massive black holes through global warming, MNRAS 425 (2012) 2974 [hep-th/1205.6467].

[19] N. Y. Gnedin, Effect of Reionization on Structure Formation in the Universe, ApJ 542 (2000) 535 [hep-th/0002151].

[20] S. Naoz, R. Barkana, The formation and gas content of high-redshift galaxies and minihaloes, MNRAS 377 (2007) 667 [hep-th/ 0612004 ].

[21] Z. Haiman, M. J. Rees, A. Loeb, $\mathrm{H}_{2}$ Cooling of Primordial Gas Triggered by UV Irradiation, ApJ 467 (1996) 522 [hep-th/9511126].

[22] M. Kuhlen, P. Madau, The first miniquasar, MNRAS 363 (2005) 1069 [hep-th/ 0506712 ].

[23] M. A. Latif, S. Bovino, T. Grassi, D. R. G. Schleicher, M. Spaans, How realistic UV spectra and $X$-rays suppress the abundance of direct collapse black holes, MNRAS 446 (2015) 3163 [hep-th/1408.3061].

[24] K. Inayoshi, T. L. Tanaka, The suppression of direct collapse black hole formation by soft X-ray irradiation, MNRAS 450 (2015) 4350 [hep-th/1411.2590].

[25] T. H. Greif, V. Bromm, P. C. Clark, S. C. O. Glover, R. J. Smith, R. S. Klessen, N. Yoshida, V. Springel, Formation and evolution of primordial protostellar systems, MNRAS 424 (2012) 399.

[26] A. Stacy, V. Bromm, Constraining the statistics of Population III binaries, MNRAS 443 (2013) 1094 [hep-th/1211.1889].

[27] J. Binney, S. Tremaine, Galactic dynamics, 1987.

[28] T. Tanaka, Z. Haiman, The Assembly of Supermassive Black Holes at High Redshifts, ApJ 696 (2009) 1798 [hep-th/0807.4702].

[29] E. C. Ostriker, Dynamical Friction in a Gaseous Medium, ApJ 513 (1999) 252 [hep-th/981032 4]. 
[30] A. Escala, R. B. Larson, P. S. Coppi, D. Mardones, The Role of Gas in the Merging of Massive Black Holes in Galactic Nuclei. I. Black Hole Merging in a Spherical Gas Cloud, ApJ 607 (2004) 765 [hep-th/0310851].

[31] F. Erwin, Low-order classical Runge-Kutta formulas with step size control and their application to some heat transfer problems, NASA Technical Report 315 (1969) 1.

[32] N. I. Shakura, R. A. Sunyaev, Black holes in binary systems. Observational appearance, A\&A 24 (1973) 337.

[33] J. Frank, A. King, D. J. Raine, Accretion Power in Astrophysics: Third Edition, 2002.

[34] J. C. Tan, C. F. McKee, The Formation of the First Stars. I. Mass Infall Rates, Accretion Disk Structure, and Protostellar Evolution, ApJ 603 (2004) 383 [hep-th/ 0307414 ].

[35] J. C. Tan, E. G. Blackman, Protostellar Disk Dynamos and Hydromagnetic Outflows in Primordial Star Formation, ApJ 603 (2004) 401 [hep-th / 0307455 ].

[36] D. Syer, C. J. Clarke, Satellites in discs: regulating the accretion luminosity, MNRAS 277 (1995) 758 [hep-th/9505021].

[37] A. Stacy, T. H. Greif, V. Bromm, The first stars: mass growth under protostellar feedback, MNRAS 422 (2012) 290 [hep-th/1109.3147].

[38] T. Hosokawa, S. Hirano, R. Kuiper, H. W. Yorke, K. Omukai, N. Yoshida, Formation of Massive Primordial Stars: Intermittent UV Feedback with Episodic Mass Accretion, ApJ 824 (2015) 119 [hep-th/1510.01407].

[39] H.-J. Grimm, M. Gilfanov, R. Sunyaev, High-mass X-ray binaries as a star formation rate indicator in distant galaxies, MNRAS 339 (2003) 793 [hep-th/ 0205371$].$

[40] M. Gilfanov, H.-J. Grimm, R. Sunyaev, $L_{\mathrm{X}}-S F R$ relation in star-forming galaxies, MNRAS 347 (2004) L57 [hep-th/0301331].

[41] M. Persic, Y. Rephaeli, V. Braito, M. Cappi, R. Della Ceca, A. Franceschini, D. E. Gruber, 2-10 keV luminosity of high-mass binaries as a gauge of ongoing star-formation rate, A\&A 419 (2004) 849 [hep-th/0402568].

[42] I. F. Mirabel, M. Dijkstra, P. Laurent, A. Loeb, J. R. Pritchard, Stellar black holes at the dawn of the universe, A\&A 528 (2011) A149 [hep-th/1102.1891].

[43] S. Mineo, M. Gilfanov, R. Sunyaev, X-ray emission from star-forming galaxies - I. High-mass X-ray binaries, MNRAS 419 (2012) 2095 [hep-th/1105.4610].

[44] K. Belczynski, V. Kalogera, F. A. Rasio, R. E. Taam, A. Zezas, T. Bulik, T. J. Maccarone, N. Ivanova, Compact Object Modeling with the StarTrack Population Synthesis Code, ApJ Supp. 174 (2008) 223 [hep-th/0511811].

[45] R. Salvaterra, F. Haardt, M. Volonteri, A. Moretti, Limits on the high redshift growth of massive black holes, A\&A 545 (2012) L6 [hep-th/1209.1095].

[46] M. S. Sipior, M. Eracleous, S. Sigurdsson, Simulating the Early Evolution of the Hard X-Ray Properties of a Young Stellar Population, (2003) hep-th/0308077.

[47] S. Migliari, R. P. Fender, Jets in neutron star X-ray binaries: a comparison with black holes, MNRAS 366 (2006) 79 [hep-th/ 0510698$].$

[48] C. Power, G. A. Wynn, C. Combet, M. I. Wilkinson, Primordial globular clusters, X-ray binaries and cosmological reionization, MNRAS 395 (2009) 1146 [hep-th/ 0902 . 1897]. 
[49] S. Repetto, M. B. Davies, S. Sigurdsson, Investigating stellar-mass black hole kicks, MNRAS $\mathbf{4 2 5}$ (2012) 2799 [hep-th/1203.3077].

[50] H.-T. Janka, Natal kicks of stellar mass black holes by asymmetric mass ejection in fallback supernovae, MNRAS 434 (2013) 1355 [hep-th/1306.0007].

[51] A. I. MacFadyen, S. E. Woosley, Collapsars: Gamma-Ray Bursts and Explosions in "Failed Supernovae”, ApJ 524 (1999) 262 [hep-th/9810274].

[52] M. Cantiello, S.-C. Yoon, N. Langer, M. Livio, Binary star progenitors of long gamma-ray bursts, $A \& A 465$ (2007) L29 [hep-th/ 0702540$].$

[53] V. Bromm, A. Loeb, High-Redshift Gamma-Ray Bursts from Population III Progenitors, ApJ 642 (2006) 382 [hep-th/0509303].

[54] S. Schanne et al., The future Gamma-Ray Burst Mission SVOM, (2010) hep-th/1005.5008.

[55] J. E. Grindlay, The Energetic X-ray Imaging Survey Telescope (EXIST), in Bulletin of the American Astronomical Society 41 (2009) American Astronomical Society Meeting Abstracts \#213 p. \#217.03.

[56] A. Mesinger, R. Perna, Z. Haiman, Constraints on the Small-Scale Power Spectrum of Density Fluctuations from High-Redshift Gamma-Ray Bursts, ApJ 623 (2005) 1 [hep-th / 0501233 ].

[57] R. Salvaterra, S. Campana, G. Chincarini, S. Covino, G. Tagliaferri, Gamma-ray bursts from the early Universe: predictions for present-day and future instruments, MNRAS 385 (2008) 189 [hep-th/0710.4280].

[58] Q. Ma, U. Maio, B. Ciardi, R. Salvaterra, PopIII signatures in the spectra of PopII/I GRBs, MNRAS 449 (2015) 3006 [hep-th / 1503 . 01118].

[59] P. P. Eggleton, Approximations to the radii of Roche lobes, ApJ 268 (1983) 368.

[60] P. C. Clark, S. C. O. Glover, R. J. Smith,T. H. Greif , R. S. Klessen , V. Bromm, The Formation and Fragmentation of Disks Around Primordial Protostars, Science 331 (2011) 1040 [hep-th/1101.5284].

[61] A. R. Basu-Zych et al., The X-Ray Star Formation Story as Told by Lyman Break Galaxies in the 4 Ms CDF-S, ApJ 762 (2013) 45 [hep-th/1210.3357].

[62] P. Kaaret, X-ray luminous binaries, metallicity, and the early Universe, MNRAS 440 (2014) L26 [hep-th/1401.5687].

[63] A. Fialkov, R. Barkana, The rich complexity of 21-cm fluctuations produced by the first stars, MNRAS 445 (2014) 213 [hep-th/1409. 3992]. 